\title{
Fractal scattering dynamics of the three-dimensional HOCI molecule
}

\author{
Yi-Der Lin, Alex M. Barr, and L. E. Reichl \\ Center for Complex Quantum Systems and Department of Physics The University of Texas at Austin, Austin, Texas 78712, USA \\ Christof Jung \\ Instituto de Ciencias Fisicas, Universidad Nacional Autonoma de Mexico Av. Universidad 1001, 62251 Cuernavaca, Mexico
}

(Received 30 May 2012; published 30 January 2013)

\begin{abstract}
We compare the 2D and 3D classical fractal scattering dynamics of $\mathrm{Cl}$ and $\mathrm{HO}$ for energies just above dissociation of the $\mathrm{HOCl}$ molecule, using a realistic potential energy surface for the $\mathrm{HOCl}$ molecule and techniques developed to analyze $3 \mathrm{D}$ chaotic scattering processes. For parameter regimes where the HO dimer initially has small vibrational energy, only small intervals of initial conditions show fractal scattering behavior and the scattering process is well described by a 2D model. For parameter regimes where the HO dimer initially has large vibrational energy, the scattering process is fully $3 \mathrm{D}$ and is dominated by fractal behavior.
\end{abstract}

DOI: 10.1103/PhysRevE.87.012917

PACS number(s): 05.45.Ac, 34.20.-b, 34.50.Ez

\section{INTRODUCTION}

Studies of fractal scattering processes have focused on systems with two degrees of freedom (2D) because in 2D they are easy to visualize [1-8] and characterize using Poincaré surfaces of section (SOS). In 2D systems it is possible to follow the flow of stable and unstable manifolds, as they form an increasingly complex network of tendrils in the phase space. These tendrils can be categorized in terms of symbolic dynamics and their fractal structure then becomes apparent.

When dealing with scattering problems with three degrees of freedom (3D), surfaces of section (SOS) become four dimensional and one cannot visualize the complexity of the scattering processes in the same way [9], although the scattering process is still dominated by the normal hyperbolic invariant manifold (NHIM) of the outer fixed point of the SOS. Jung et al. [10] have shown that in some parameter regimes, a 3D system can be viewed as a 2D system with a weakly coupled third degree of freedom for which an approximate conserved quantity (exact for the 2D system) exists. The four-dimensional surface of section can then be viewed as a continuous "stack" of two-dimensional surfaces of section. The outer fixed point of the "stack" of 2D systems forms a continuous NHIM [9,11-13]. They find that this NHIM and its homoclinic tangle appear to remain structurally robust in the presence of a small perturbation.

In subsequent sections, we analyze the scattering dynamics of $\mathrm{Cl}$ and $\mathrm{HO}$ just above the dissociation energy of the $\mathrm{HOCl}$ molecule. Our analysis of $\mathrm{Cl}-\mathrm{HO}$ scattering dynamics uses a potential energy surface (PES), constructed by Weiss et al. $[14,15]$, that realistically governs the dynamics of the bound $\mathrm{HOCl}$ molecule and the $\mathrm{Cl}-\mathrm{HO}$ scattering system for an interval of energies just above dissociation of $\mathrm{Cl}$ from $\mathrm{HO}$. For the 3D Cl-HO scattering process, we find behavior similar to that observed by Jung et al. [10], with the vibrational degree of freedom of $\mathrm{HO}$ playing the role of a weakly coupled third degree of freedom (at least for some parameter regimes). For a given initial energy of $\mathrm{Cl}$ and $\mathrm{HO}$, the initial conditions of both objects can be specified in terms of two initial "phases" (which we define below). Evidence of the robustness of the NHIM in the $\mathrm{Cl}-\mathrm{HO}$ system can be seen in plots of the magnitude of the outgoing momentum of the scattered $\mathrm{Cl}$ atom as a function of the initial phases. This provides a "landscape" that shows a range of initial phases for which the scattering function (outgoing momentum versus initial phases) appears to have fractal structure. This fractal structure is formed by the intersection of the stable manifold of the NHIM (which extends into the asymptotic region) with the torus formed by the initial phases of $\mathrm{Cl}$ and $\mathrm{HO}$ in the asymptotic region. When a $\mathrm{Cl}$ trajectory has an initial phase that lies on the NHIM in the asymptotic region, the subsequent trajectory of $\mathrm{Cl}$ will have an infinite delay time and the outgoing $\mathrm{Cl}$ momentum will approach zero in the asymptotic region as the trajectory approaches the outer fixed point (which by definition has zero momentum). These singular points form a fractal structure which is an intrinsic property of the molecule, and all other dynamical quantities will exhibit this same fractal behavior.

We begin in Sec. II with a description of the Hamiltonian and the potential energy surface for the 3D HOCl molecule. In Sec. III, we describe the scattering dynamics. In Sec. IV, we compare the scattering dynamics of the 3D and 2D models of the $\mathrm{HOCl}$ molecule for the case when the $\mathrm{HO}$ dimer initially has a small amount of vibrational energy. In Sec. V, we show the effect on the scattering phase space of starting the scattering process with the $\mathrm{HO}$ dimer in a fairly high energy vibrational state. In Sec. VI, we make some concluding remarks.

\section{THE 3D MODEL OF HOCL}

In its bound configuration, the $\mathrm{HOCl}$ molecule consists of $\mathrm{H}, \mathrm{O}$, and $\mathrm{Cl}$ atoms, with masses $m_{\mathrm{H}}, m_{\mathrm{O}}$, and $m_{\mathrm{Cl}}$, respectively. The molecule dissociates into a free $\mathrm{Cl}$ atom and a bound $\mathrm{HO}$ molecule above $E=20312 \mathrm{~cm}^{-1}$. The classical dynamics of the $\mathrm{HOCl}$ system has been discussed in Ref. [7] for a 2D version of the molecule in which the HO bond is held fixed. We here relax that constraint on the HO bond and consider the 3D dynamics of the molecule. As in Ref. [7], we introduce laboratory coordinates $\left(x^{\prime}, y^{\prime}, z^{\prime}\right)$ and body-fixed coordinates $(x, y, z)$ whose origin is the center of mass of the molecule. The total angular momentum of the molecule, $\mathbf{L}_{\text {tot }}$, is conserved. We assume that all of the dynamics occurs in the $\left(x^{\prime}, z^{\prime}\right)$ and $(x, z)$ planes and that any angular momentum vectors generated by internal rotations of the molecule lie along parallel $y^{\prime}$ and 


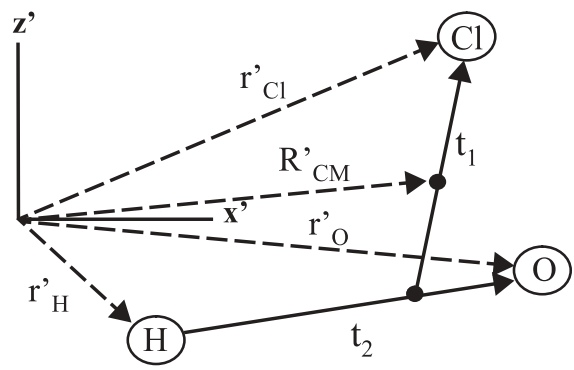

(a)

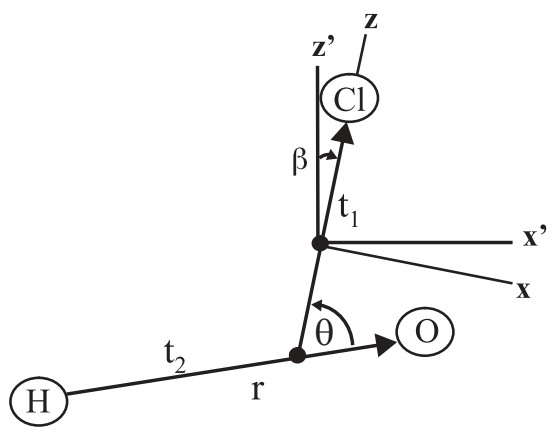

(b)

FIG. 1. (a) Relationship between laboratory frame coordinates and Jacobi vectors $\mathbf{t}_{1}$ and $\mathbf{t}_{2}$ and $\theta$. (b) Body-fixed and laboratory axes differ by the angle $\beta$ so $\mathbf{t}_{1}$ always lies along the body $z$ axis.

$y$ axes in the laboratory and body-fixed frames, respectively. A sketch of $\mathrm{HOCl}$ relative to the laboratory and body frames is shown in Fig. 1.

Let $\mathbf{t}_{1}$ be a vector of length $R$ that connects the center of mass of $\mathrm{HO}$ to $\mathrm{Cl}$ and $\mathbf{t}_{2}$ a vector of length $r$ that connects $\mathrm{H}$ to $\mathrm{O}$. The angle between $\mathbf{t}_{1}$ and $\mathbf{t}_{2}$ is $\theta$ where $\theta=0$ for the linear configuration $\mathrm{H}-\mathrm{O}-\mathrm{Cl}$. The center of mass of the molecule lies along $\mathbf{t}_{1}$ a distance $m_{d} R / M$ from the $\mathrm{Cl}$ atom, where $m_{d}=m_{\mathrm{O}}+m_{\mathrm{H}}$ and $M=m_{\mathrm{Cl}}+m_{\mathrm{O}}+m_{\mathrm{H}}$. Let $\mathbf{r}_{\mathrm{Cl}}^{\prime}, \mathbf{r}_{\mathrm{H}}^{\prime}$, and $\mathbf{r}_{\mathrm{O}}^{\prime}$ denote the displacement of the $\mathrm{Cl}, \mathrm{H}$, and $\mathrm{O}$ atoms from the laboratory frame origin. Let $\mathbf{R}_{\mathrm{c} . \mathrm{m} .}=\left(m_{\mathrm{Cl}} \mathbf{r}_{\mathrm{Cl}}^{\prime}+m_{\mathrm{O}} \mathbf{r}_{\mathrm{O}}^{\prime}+\right.$ $\left.m_{\mathrm{H}} \mathbf{r}_{\mathrm{H}}^{\prime}\right) / M$ denote the displacement of the center of mass of $\mathrm{HOCl}$ from the laboratory frame origin. The kinetic energy then is given by

$$
T=\frac{M}{2} \dot{\mathbf{R}}_{\text {c.m. }}^{2}+\frac{\mu_{1}}{2} \dot{\mathbf{t}}_{1}^{2}+\frac{\mu_{2}}{2} \dot{\mathbf{t}}_{2}^{2}
$$

and the total angular momentum is given by

$$
\mathbf{L}_{\text {tot }}=\mu_{1} \mathbf{t}_{1} \times \dot{\mathbf{t}}_{1}+\mu_{2} \mathbf{t}_{2} \times \dot{\mathbf{t}}_{2},
$$

where $\mu_{1}=\frac{m_{\mathrm{C}} m_{d}}{M}=\frac{595}{52} u$ and $\mu_{2}=\frac{m_{\mathrm{H}} m_{\mathrm{O}}}{m_{d}}=\frac{16}{17} u$ (u the atomic mass unit).

We will assume that $\mathbf{t}_{1}$ lies along the body $z$ axis and that the body $y$ axis is directed perpendicularly to the plane of the molecule. We can then write $\mathbf{t}_{1}=R \hat{\mathbf{z}}$ and $\mathbf{t}_{2}=r \sin (\theta) \hat{\mathbf{x}}+$ $r \cos (\theta) \hat{\mathbf{z}}$. We further assume that the body frame $(x, z)$ axes make an angle $\beta$ with respect to the laboratory frame $\left(x^{\prime}, z^{\prime}\right)$ axes so if the two frames rotate relative to one another, the angular velocity of rotation is $\dot{\beta} \hat{\mathbf{y}}$.
The Hamiltonian for the $\mathrm{HO}+\mathrm{Cl}$ system (dropping the center-of-mass motion) can then be written in the form

$$
\begin{aligned}
H= & \frac{p_{R}^{2}}{2 \mu_{1}}+\frac{p_{r}^{2}}{2 \mu_{2}}+\frac{p_{\theta}^{2}}{2 \mu_{2} r^{2}}+\frac{p_{\theta}^{2}}{2 \mu_{1} R^{2}} \\
& +\frac{p_{\beta}^{2}}{2 \mu_{1} R^{2}}-\frac{p_{\theta} p_{\beta}}{\mu R^{2}}+D_{e} V(R, \theta, r)=E,
\end{aligned}
$$

where $E$ is the total energy. The quantity $D_{e} V(R, \theta, r)$ is the potential energy and $D_{e}=20312.3 \mathrm{~cm}^{-1}=2.518 \mathrm{eV}$ is the energy at which $\mathrm{Cl}$ dissociates from HO. The canonical momenta $p_{R}, p_{r}, p_{\theta}$, and $p_{\beta}$ can be written $p_{R}=\mu_{1} \dot{R}, p_{r}=$ $\mu_{2} \dot{r}, p_{\theta}=\mu_{2} r^{2}(\dot{\theta}+\dot{\beta})$, and $p_{\beta}=\mu_{2} r^{2}(\dot{\theta}+\dot{\beta})+\mu_{1} R^{2} \dot{\beta}$. The total angular momentum of the molecule is then given by

$$
\mathbf{L}_{\text {tot }}=\mathbf{L}_{1}+\mathbf{L}_{2}=p_{\beta} \hat{\mathbf{y}}
$$

where

$$
\begin{aligned}
& \mathbf{L}_{1}=\mu_{1} \mathbf{t}_{1} \times \dot{\mathbf{t}}_{1}=\mu_{1} R^{2} \dot{\beta} \hat{\mathbf{y}} \text { and } \\
& \mathbf{L}_{2}=\mu_{2} \mathbf{t}_{2} \times \dot{\mathbf{t}}_{2}=\mu_{2} r^{2}(\dot{\theta}+\dot{\beta}) \hat{\mathbf{y}} .
\end{aligned}
$$

In subsequent sections, we shall examine the scattering of $\mathrm{Cl}$ from $\mathrm{HO}$ for a subset of initial conditions for which $\mathbf{L}_{\text {tot }}=$ 0 . We accomplish this by requiring that $p_{\beta}=0$ in all initial trajectories. Since total angular momentum is conserved for the $\mathrm{HOCl}$ system, then $p_{\beta}=0$ for the entire scattering process. Note, however, that $\mathbf{L}_{1}$ and $\mathbf{L}_{2}$ need not be zero so $\mathrm{Cl}$ and $\mathrm{HO}$ can rotate relative to one another.

It is useful to write the above quantities in terms of dimensionless units (d.u.). We parametrize all energies in units of $D_{e}$, lengths in units of the Bohr radius $a_{B}=5.2917 \times 10^{-11} \mathrm{~m}$, and angular momenta in terms of Planck's constant $\hbar=$ $1.05457 \times 10^{-34} \mathrm{~J} \mathrm{~s}$. Then $H=D_{e} H^{\prime}, E=D_{e} E^{\prime}, R=a_{B} R^{\prime}$, $r=a_{B} r^{\prime}, p_{R}=\frac{\hbar}{a_{B}} p_{R}^{\prime}, p_{r}=\frac{\hbar}{a_{B}} p_{r}^{\prime}, p_{\theta}=\hbar p_{\theta}^{\prime}, p_{\beta}=\hbar p_{\beta}^{\prime}$, and time $t=\frac{\hbar}{D_{e}} t^{\prime}$, where primed quantities are dimensionless. If we now drop the primes on dimensionless quantities, the (dimensionless) Hamiltonian takes the form

$$
\begin{aligned}
H= & \frac{p_{R}^{2}}{2 \delta_{1}}+\frac{p_{r}^{2}}{2 \delta_{2}}+\frac{p_{\theta}^{2}}{2 \delta_{2} r^{2}}+\frac{p_{\theta}^{2}}{2 \delta_{1} R^{2}} \\
& +\frac{p_{\beta}^{2}}{2 \delta_{1} R^{2}}-\frac{p_{\theta} p_{\beta}}{\delta_{1} R^{2}}+V(R, \theta, r)=E,
\end{aligned}
$$

where $\quad \delta_{1}=\frac{\mu_{1} D_{e} a_{B}^{2}}{\hbar^{2}}=1930.3$ d.u. $\quad$ and $\quad \delta_{2}=\frac{\mu_{2} D_{e} a_{B}^{2}}{\hbar^{2}}=$ 158.79 d.u. Since we are considering scattering dynamics for the case $p_{\beta}=0$, this reduces to a system with three degrees of freedom.

We use the potential energy surface (PES) constructed by Weiss et al. $[14,15]$ which governs the dynamics of the HOCl molecule and the $\mathrm{Cl}$ and $\mathrm{HO}$ system for a region of energies just above dissociation of $\mathrm{Cl}$ from $\mathrm{HO}$. This PES can be written in the form

$$
V\left(R_{\mathrm{OCl}}, R_{\mathrm{HO}}, R_{\mathrm{ClH}}\right)=V_{I}\left(R_{\mathrm{OCl}}, R_{\mathrm{HO}}, R_{\mathrm{ClH}}\right)+V_{\mathrm{HO}}\left(R_{\mathrm{HO}}\right) .
$$

where $R_{\mathrm{OCl}}, R_{\mathrm{HO}}$, and $R_{\mathrm{ClH}}$ are the distances between $\mathrm{O}$ and $\mathrm{Cl}, \mathrm{H}$ and $\mathrm{O}$, and $\mathrm{Cl}$ and $\mathrm{H}$, respectively (in dimensionless 
units), and

$$
V_{\mathrm{HO}}\left(R_{\mathrm{HO}}\right)=1.835\left[1-e^{-\beta_{\mathrm{HO}}\left(R_{\mathrm{HO}}-\bar{R}_{\mathrm{HO}}\right)}\right]^{2},
$$

is the potential energy of the HO system in the asymptotic region with $\beta_{\mathrm{HO}}=1.2139$ d.u. and $\bar{R}_{\mathrm{HO}}=1.8323$ d.u. The potential energy $V_{I}\left(R_{\mathrm{OCl}}, R_{\mathrm{HO}}, R_{\mathrm{ClH}}\right)$ is the interaction energy of $\mathrm{Cl}$ and $\mathrm{HO}$ and has the form

$$
\begin{aligned}
V_{I}( & \left.R_{\mathrm{OCl}}, R_{\mathrm{HO}}, R_{\mathrm{ClH}}\right) \\
= & \frac{1}{2}\left[1+\tanh \left(6-R_{\mathrm{ClO}}\right)\right] \\
& \times \sum_{i=0}^{7} \sum_{j=0}^{7} \sum_{\ell=0}^{7} a_{i, j, \ell} g_{i}\left(R_{\mathrm{HO}}\right) h_{j}\left(R_{\mathrm{OCl}}\right) d_{\ell}\left(R_{\mathrm{ClH}}\right),
\end{aligned}
$$

where

$$
\begin{aligned}
g_{i}\left(R_{\mathrm{HO}}\right) & =\left[1-e^{-k_{\mathrm{HO}}\left(R_{\mathrm{HO}}-\bar{R}_{\mathrm{HO}}\right)}\right]^{i}, \\
h_{j}\left(R_{\mathrm{OCl}}\right) & =\left[1-e^{-k_{\mathrm{OCl}}\left(R_{\mathrm{OCl}}-\bar{R}_{\mathrm{OCl}}\right)}\right]^{j+1}, \\
d_{\ell}\left(R_{\mathrm{ClH}}\right) & =\left[1-e^{-k_{\mathrm{ClH}}\left(R_{\mathrm{ClH}}-\bar{R}_{\mathrm{ClH}}\right)}\right]^{\ell},
\end{aligned}
$$

$\bar{R}_{\mathrm{HO}}=1.85$ d.u., $\bar{R}_{\mathrm{OCl}}=3.2$ d.u., $\bar{R}_{\mathrm{ClH}}=4$ d.u., $k_{\mathrm{HO}}=$ 0.3 d.u., $k_{\mathrm{OCl}}=0.8$ d.u., and $k_{\mathrm{ClH}}=0.1$ d.u. Note that $R_{\mathrm{HO}}=$ $r$ and the distances $R_{\mathrm{OCl}}, R_{\mathrm{HO}}$, and $R_{\mathrm{ClH}}$ are related to $R, r$, and $\theta$ by the triangle equations

$$
R_{\mathrm{OCl}}=\sqrt{\left(\frac{m_{H}}{m_{d}}\right)^{2} r^{2}+R^{2}-2\left(\frac{m_{H}}{m_{d}}\right) r R \cos (\theta)}
$$

and

$$
R_{\mathrm{ClH}}=\sqrt{\left(\frac{m_{O}}{m_{d}}\right)^{2} r^{2}+R^{2}+2\left(\frac{m_{O}}{m_{d}}\right) r R \cos (\theta)} .
$$

The ground-state (lowest bound state) energy for this system is $E=0$ d.u. The dissociation energy for $\mathrm{Cl}$ from $\mathrm{HO}$, when rotation and vibration energy of $\mathrm{HO}$ is zero, is $E=D_{e}=$ 1.0 d.u. The $2 \mathrm{D}$ model of $\mathrm{HOCl}$ is obtained by setting $r=\bar{R}_{\mathrm{HO}}$ and $p_{r}=0$ in Eq. (6) and $R_{\mathrm{HO}}=\bar{R}_{\mathrm{HO}}$ in Eqs. (8) and (10).

\section{SCATTERING DYNAMICS}

In the 3D model of $\mathrm{HOCl}$, the energy and the total angular momentum of the $\mathrm{HOCl}$ system are constants of the motion, and both the $\mathrm{HO}$ rotation and vibration participate in the dynamics of the scattering process. For energies just above dissociation, $\mathrm{Cl}$ impinges on the $\mathrm{HO}$ complex and scatters from it, leaving both the $\mathrm{Cl}$ atom and the $\mathrm{HO}$ complex in an altered state. In order to study this scattering process, we must specify initial conditions for both the $\mathrm{Cl}$ atom and the $\mathrm{HO}$ complex.

We can distribute the initial total energy $E$ (which is conserved) between the incident energy of $\mathrm{Cl}, E_{\mathrm{Cl}}=\frac{p_{R}^{2}}{2 \delta_{1}}$, and the rotational and vibration energies of $\mathrm{HO}, E_{\mathrm{rot}}$ and $E_{\mathrm{vib}}$, so $E=E_{\mathrm{Cl}}+E_{\mathrm{rot}}+E_{\mathrm{vib}}$. The initial value of $E_{\mathrm{Cl}}$ fixes the initial value of $p_{R}$. The remaining energy is distributed between $E_{\text {rot }}$ and $E_{\mathrm{vib}}$. The angular momentum of $\mathrm{HO}$ relative to its center of mass is given by $\mathbf{L}_{2}=p_{\theta} \hat{\mathbf{y}}$. For a given total energy $E$, in the asymptotic region $\left(R_{\text {in }}=R_{\text {out }}=12\right.$ d.u. $)$, we can specify a range of initial values of $E_{\mathrm{Cl}}$. We also specify a range of initial values for the angular position $\chi=\theta$ and vibrational phase $\psi$ in the asymptotic region. Each initial condition therefore can be labeled uniquely by $\left(E, p_{R}, p_{\theta}, \chi, \psi\right)$.

The phase $\chi=\theta$ is the initial angular position of the $\mathrm{HO}$ dimer at $R_{\text {in }}=12$ d.u. The phase $\psi$ is determined by the initial vibrational configuration of $\mathrm{HO}$ in the asymptotic region and ranges over all possible initial configurations of $\mathrm{HO}$ at its given initial energy. It is determined as follows. In the asymptotic regime the Hamiltonian of $\mathrm{HO}$ is given by

$$
H_{\mathrm{HO}}=\frac{p_{r}^{2}}{2 \delta_{2}}+\frac{p_{\theta}^{2}}{2 \delta_{2} r^{2}}+V_{\mathrm{HO}}(r)=E_{\mathrm{rot}}+E_{\mathrm{vib}}=E_{\mathrm{HO}} .
$$

The value of $p_{\theta}$ and the total energy of $\mathrm{HO}$ are fixed, and the initial values of $\left(p_{r}, r\right)$ are allowed to range over one complete oscillation of the $\mathrm{HO}$ vibration at that total energy. The phase $\psi$ ranges over the interval $0 \leqslant \psi \leqslant 2 \pi$ as $\mathrm{HO}$ goes through one complete oscillation.

For a given value of total energy $E$, we can plot values of $p_{R}$ and $p_{\theta}$ after the scattering process has occurred for ranges of initial phases $0 \leqslant \chi \leqslant 2 \pi$ and $0 \leqslant \psi \leqslant 2 \pi$ and different incident $\mathrm{Cl}$ energies $E_{\mathrm{Cl}}$. In subsequent sections, we analyze scattering properties for a subspace of the scattering process with total angular momentum zero and total energies in the interval 1.034 d.u. $\leqslant E \leqslant 1.231$ d.u. $\left(21000 \mathrm{~cm}^{-1} \leqslant E \leqslant\right.$ $25000 \mathrm{~cm}^{-1}$ ). The PES that we use was developed to represent the dynamics of $\mathrm{HOCl}$ above dissociation for these and slightly higher energies. The subspace with total angular momentum zero gives us a good picture of the nature of the scattering processes. We do not expect it to change significantly for nonzero total angular momentum.

In the scattering process, the total energy is distributed between the rotational and vibrational energy of $\mathrm{HO}$ and the incident energy of $\mathrm{Cl}$ in different ways. If $\mathrm{Cl}$ approaches $\mathrm{HO}$ with its momentum directed along a line through the center of mass of $\mathrm{HO}$, then its impact parameter is zero and $\mathrm{HO}$ does not rotate. If $\mathrm{Cl}$ approaches $\mathrm{HO}$ with a nonzero impact parameter relative to the center of mass of $\mathrm{HO}$, then it will have angular momentum and the $\mathrm{HO}$ molecule will rotate in such a manner that its rotational angular momentum cancels the angular momentum of the $\mathrm{Cl}$ atom. In the energy interval 1.034 d.u. $\leqslant E \leqslant 1.231$ d.u., HO remains a bound molecule and acts dynamically like an anharmonic oscillator that is coupled into the scattering between the center of mass of $\mathrm{HO}$ and the $\mathrm{Cl}$ atom.

\section{2D VERSUS 3D MODELS OF HOC}

The HO vibration is very stiff compared to that of the $\mathrm{HO}-\mathrm{Cl}$ vibration. This fact has formed the basis of two papers that have modeled the dynamic properties of the $\mathrm{HOCl}$ molecule as a $2 \mathrm{D}$ system, holding the $\mathrm{HO}$ vibration fixed $[7,16]$. In this and subsequent sections, we compare the scattering dynamics of the 2D and 3D models of $\mathrm{HOCl}$ at energies above dissociation.

In Fig. 2, we compare the scattering behavior of $p_{R}$ and $p_{\theta}$ for the $3 \mathrm{D}$ case, when the total energy of the $\mathrm{Cl}+\mathrm{HO}$ system is $E=1.231$ d.u. $=25000 \mathrm{~cm}^{-1}$, the $\mathrm{HO}$ molecule has an initial total energy $E_{\mathrm{HO}}=0.03282 \mathrm{~d} . \mathrm{u} .=667 \mathrm{~cm}^{-1}$, and an initial angular momentum $p_{\theta}=5.92 \mathrm{~d}$.u. For these parameters, the initial energy of $\mathrm{HO}$ is almost entirely rotational. The initial range of oscillation of $\mathrm{HO}$ is 1.8333 d.u. $\leqslant r \leqslant 1.8446$ d.u., 


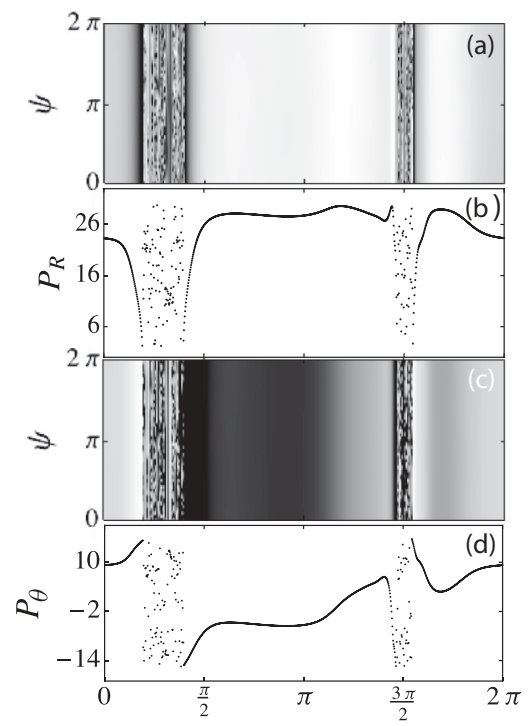

FIG. 2. The scattering dynamics for the $3 \mathrm{D}$ molecule when total energy of the $\mathrm{Cl}+\mathrm{H}-\mathrm{O}$ system is $E=1.231$ d.u. $=25000 \mathrm{~cm}^{-1}$. The HO dimer has an initial total energy $E_{\mathrm{HO}}=0.03261 .231 \mathrm{~d} . \mathrm{u} .=$ $667 \mathrm{~cm}^{-1}$. The initial value of $p_{\theta}$ is $p_{\theta}=5.92 \mathrm{~d}$.u. With this value of $p_{\theta}, \mathrm{HO}$ is essentially in its vibrational ground state initially. (a) A contour plot of scattered values of $p_{R}$ as a function of $\psi$ (vertical axis) and $\chi$ (horizontal axis). (b) A plot of scattered values of $p_{R}$ as a function of $\chi$ for $\psi=\pi$. Intervals of continuity and chaos are clearly seen. (c) A contour plot of scattered values of $p_{\theta}$ as a function of $\psi$ (vertical axis) and $\chi$ (horizontal axis). (d) A plot of scattered values of $p_{\theta}$ as a function of $\chi$ for $\psi=\pi$. The intervals of continuity and of discontinuity are the same as those of $p_{R}$, indicating that they are an intrinsic property of the molecule. All plots contain 1000 data points along the $\chi$ axis and 50 data points along the $\psi$ axis. The grayscale in (a) and (c) ranges from 0 to 32, with 32 the lightest shade. Values of $p_{R}$ and $p_{\theta}$ at the darkest and lightest points in (a) and (c) can be read from (b) and (d), respectively. ( $p_{R}$ and $p_{\theta}$ in dimensionless units and $\chi$ and $\psi$ in radians.)

so $\mathrm{HO}$ is close to its minimum vibration energy. Figures 2(a) and 2(c) show contour plots of scattered values of $p_{R}$ and $p_{\theta}$, respectively, as a function of $\psi$ (vertical axis) and $\chi$ (horizontal axis). Figures 2(b) and 2(d) show plots of scattered values of $p_{R}$ and $p_{\theta}$, respectively, as a function of $\chi$ for fixed $\psi=\pi$ (for $\psi=\pi$, HO is initially at the turning point with minimum value of $r$ ). All of the plots in Fig. 2 contain 1000 data points for $\chi$ and 50 data points for $\psi$. Figures 2(b) and 2(d) show that the scattering data contain large ranges of $\chi$ with intervals of continuity and two shorter ranges of $\chi$ that contain a fractal distribution (of measure zero) of singularities. These are actually points of intersection of the stable manifold of the outer NHIM with the set of initial conditions. The fractal set of singularities form the boundaries of a complimentary fractal set of intervals of continuity, so the scattering function becomes discontinuous in this region. In Fig. 2, these intervals of continuity and singular points appear to be approximately independent of $\psi$ (the initial configuration of $\mathrm{HO}$ ). This indicates that the scattering dynamics is well approximated by the 2D model used in Refs. [7,16] in which the HO vibration is held fixed. The fractal nature of the scattering process is intrinsic to the $\mathrm{Cl}-\mathrm{HO}$ system above dissociation and the same
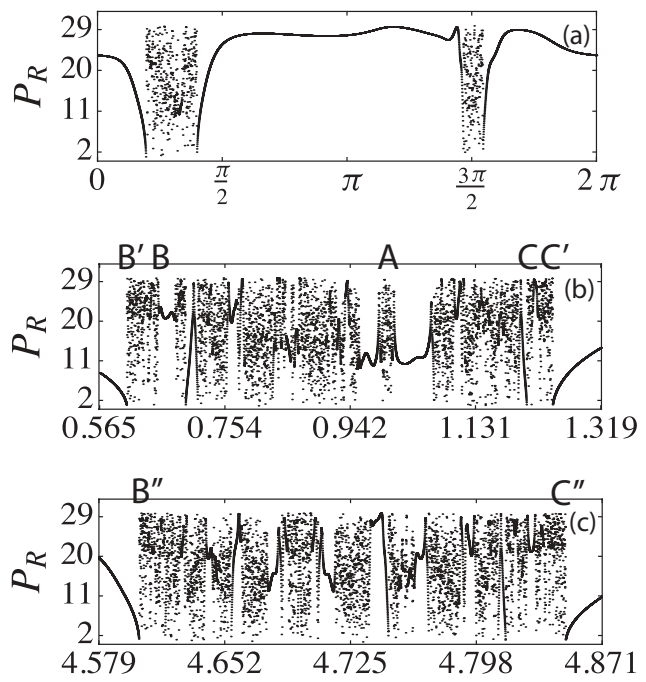

FIG. 3. Scattering dynamics of the 3D Cl-HO system. (a) Magnification of Fig. 2(b) (5000 data points for $\chi$ ). (b) Magnification of the left discontinuous region of Fig. 3(a). (c) Magnification of the right discontinuous region of Fig. 3(a). ( $p_{R}$ in dimensionless units and $\chi$ in radians.)

fractal structure will appear in all scattering functions. This can be seen in the Figs. 2(a) and 2(c), which show asymptotic values of $p_{R}$ and $p_{\theta}$ after the scattering process. The fractal structure of these plots is identical.

In Fig. 3(a), we again plot $p_{R}$ versus $\chi$, as in Fig. 2(b), but now with 5000 data points for $\chi$, so more of the fractal structure emerges. In Figs. 3(b) and 3(c), we enlarge the fractal regions on the left and right of Fig. 3(a). In Fig. 4 we show plots of $p_{R}$ versus $\chi$ for the 2D model of $\mathrm{HOCl}$ with the $\mathrm{HO}$ bond held fixed at its equilibrium displacement. Comparing
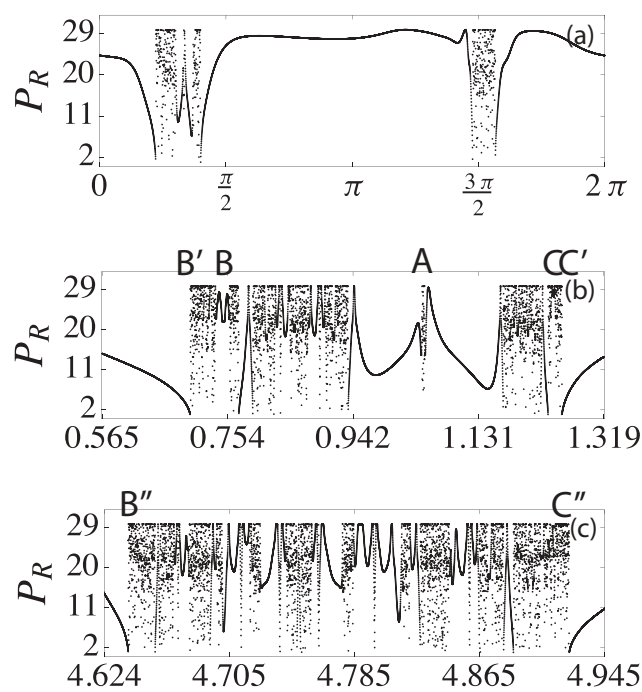

FIG. 4. Scattering dynamics of the $2 \mathrm{D} \mathrm{Cl-HO}$ system. (a) A plot of $p_{R}$ versus $\chi$ for the case is which the bond length of the HO dimer is held fixed at the equilibrium value $r_{\mathrm{eq}}=1.8389$ d.u., $p_{\theta}=5.92$ d.u., and the total energy is fixed at $E=1.231 \mathrm{~d}$.u. $=25000 \mathrm{~cm}^{-1}(5000$ data points for $\chi$ ). (b) Magnification of left discontinuous region in Fig. 4(a). (c) Magnification of the right discontinuous region in Fig. 4(a). ( $p_{R}$ in dimensionless units and $\chi$ in radians.) 
Figs. 3(a) and 4(a), we see that the scattering dynamics of the two cases is very similar, although there are also small differences. In both Fig. 3(b) and Fig. 4(b) there are "mirror" points in the discontinuous regions, located directly under the letters $\mathrm{B}, \mathrm{A}$, and $\mathrm{C}$. The structures of local regions on either side of these mirror points are approximate mirror images of each other, although the right-hand side is compressed relative to the left-hand side. The large scale similarities between the plots in Figs. 3 and 4 indicate that the 2D model of $\mathrm{HOCl}$ is fairly good at reproducing the regions of continuity and discontinuity in the 3D dynamics, as long as the $\mathrm{HO}$ vibration is close to its minimum value initially. It therefore appears that a 2D model of $\mathrm{HOCl}$ dynamics gives a good approximation to the 3D HOCl dynamics.

Although the plots in Figs. 3 and 4 are very similar, it is clear that small parts of the intervals of continuity in the 2D case contain singular points and become discontinuous in the $3 \mathrm{D}$ case. This is seen most clearly when comparing the mirror points $\mathrm{B}$ and $\mathrm{A}$ in Figs. 3(b) and 4(b), respectively. These mirrorlike structures repeat and can be found embedded locally in these plots as one goes to ever finer scales in the phase space, so there appears to be a fractal structure embedded in the phase space. Using the "box-counting" technique, we have computed the fractal dimension of the intervals of discontinuity that appear to the left- and right-hand sides of Figs. 3(a) and 4(a). For the interval of discontinuity on the left [enlarged in Figs. 3(b) and 4(b)], we find that the intervals of discontinuity for $3 \mathrm{D}$ have a fractal dimension of 0.88 , while the intervals of discontinuity for 2D have a fractal dimension of 0.80 . For the interval of discontinuity on the right [enlarged in Figs. 3(c) and 4(c)], we find that the intervals of discontinuity for 3D have fractal dimension of 0.91 , while the intervals of discontinuity for $2 \mathrm{D}$ have a fractal dimension of 0.86 .

In Fig. 5, we show the time it takes for $\mathrm{Cl}$ to leave the asymptotic region at $R=12 \mathrm{~d}$.u., interact with $\mathrm{HO}$, and finally return to the asymptotic region at $R=12 \mathrm{~d}$.u. for a range of values of initial phase $\chi$ in the neighborhood of the mirror point at B. Note that Fig. 5 shows the running times $\tau$ for $\mathrm{Cl}$ [time for $\mathrm{Cl}$ to "run" from its initial position at $R=12$ d.u., interact with HO (delay time), and then "run" back to its initial position]. The "running time" for a free particle to travel a distance $2 R$ is
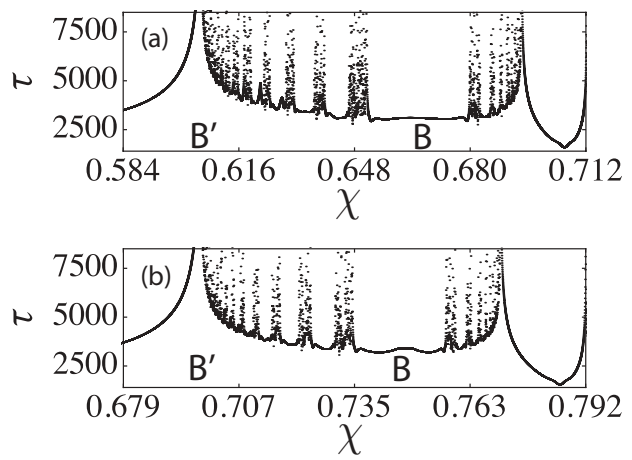

FIG. 5. Running time plots for values of $\chi$ in the neighborhood of the mirror point $\mathrm{B}$. Note that these plots contain the time for $\mathrm{Cl}$ to travel from $R=12$ d.u. to the reaction region, then interact with $\mathrm{HO}$ and return to $R=12$ d.u. (a) $3 \mathrm{D}$ molecule. (b) $2 \mathrm{D}$ molecule. ( $\tau$ in dimensionless units and $\chi$ in radians.)
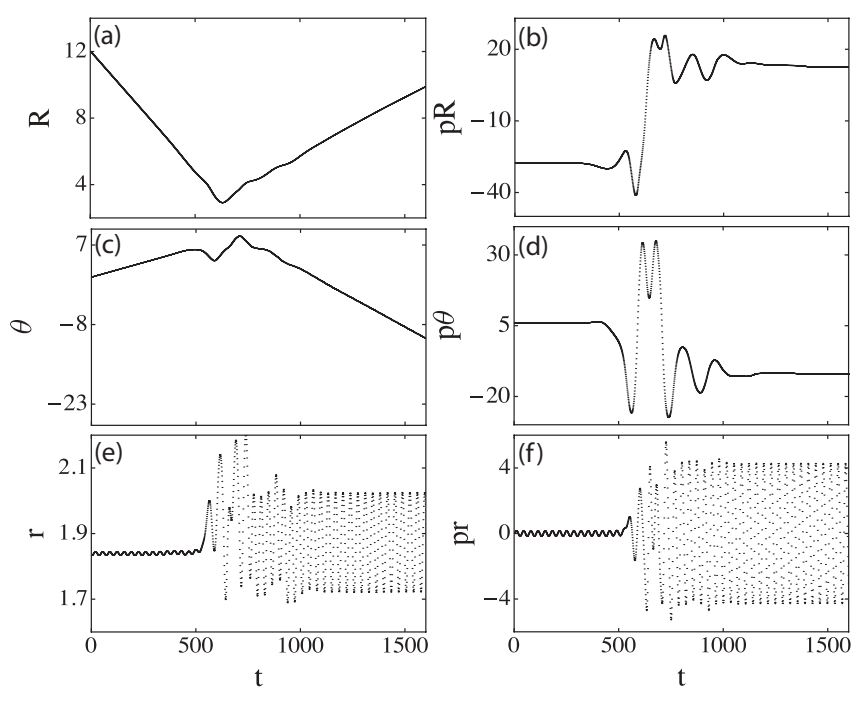

FIG. 6. The scattering dynamics of a single trajectory for $0 \leqslant$ $t \leqslant 1600$, for initial conditions $E=1.231$ d.u., $\psi=\pi$, and $\chi=$ 0.967613. (a) $R(t)$ versus $t$. (b) $p_{R}(t)$ versus $t$. (c) $\theta(t)$ versus $t$. (d) $p_{\theta}(t)$ versus $t$. (e) $r(t)$ versus $t$. (f) $p_{r}(t)$ versus $t$. $\left(p_{R}, p_{\theta}, p_{r}, R\right.$, $r$, and $t$ in dimensionless units and $\theta$ in radians.)

approximately 3000 d.u. The running time for the 3D case for $\psi=\pi$ and 0.584 d.u. $\leqslant \chi \leqslant 0.712$ d.u. is shown in Fig. 5(a). In Fig. 5(b) we show the running time for the 2D case for the interval 0.679 d.u. $\leqslant \chi \leqslant 0.792$ d.u. (The 3D plot are shifted in $\chi$, relative to the $2 \mathrm{D}$ plot, because a small amount of initial energy is shifted from the $\mathrm{Cl}$ angular momentum to the $\mathrm{HO}$ vibration in the $3 \mathrm{D}$ case.) These plots are again very similar, and they show the same fractal structure as the corresponding plots for $p_{R}$ versus $\chi$. However, the running time plots do have some differences which show that the $\mathrm{HO}$ vibration can become involved in the scattering process, even if it has very little energy initially.

It is interesting to compare trajectories from the regions of continuity and discontinuity for the 3D case. In Fig. 6, we show collision dynamics of a single trajectory taken from the region of continuity near the dominant mirror point A in Fig. 3(b). Figures 6(a) and 6(b) show $R$ and $p_{R}$ versus $t$, Figs. 6(c) and 6(d) show $\theta$ and $p_{\theta}$ versus $t$, and Figs. 6(e) and 6(f) show $r$ and $p_{r}$ versus $t$. The collision takes a very short time. The $\mathrm{Cl}$ atom bounces off of $\mathrm{HO}$ and is scattered back into the asymptotic region, after having given some of its incident energy to the rotation and vibration modes of $\mathrm{HO}$. The direction of rotation of $\mathrm{HO}$ is reversed and the vibration energy of $\mathrm{HO}$ is increased by the collision. The time between entering and leaving the collision is approximately $\Delta t_{\text {coll }}=600 \mathrm{~d}$.u.

In Fig. 7, we show the collision dynamics of a single trajectory taken from the region of discontinuity around the main mirror point $\mathrm{A}$ in Fig. 3(b). For this case, the $\mathrm{Cl}$ atom remains in the reaction region for a very long time before it is ejected back into the asymptotic region. For this trajectory, the time between entering and leaving the reaction region is approximately $\Delta t_{\text {coll }}=1015800 \mathrm{~d}$.u. Figures 7 (a) and 7(b) show the behavior of $R$ versus $t$ as the $\mathrm{Cl}$ enters and leaves, respectively, the reaction region. Similarly, Figs. 7(c) and 7(d) show plots of $\theta$ versus $t$, and Figs. 7(e) and 7(f) show plots of $r$ versus $t$, as $\mathrm{Cl}$ enters and leaves, respectively, the reaction 

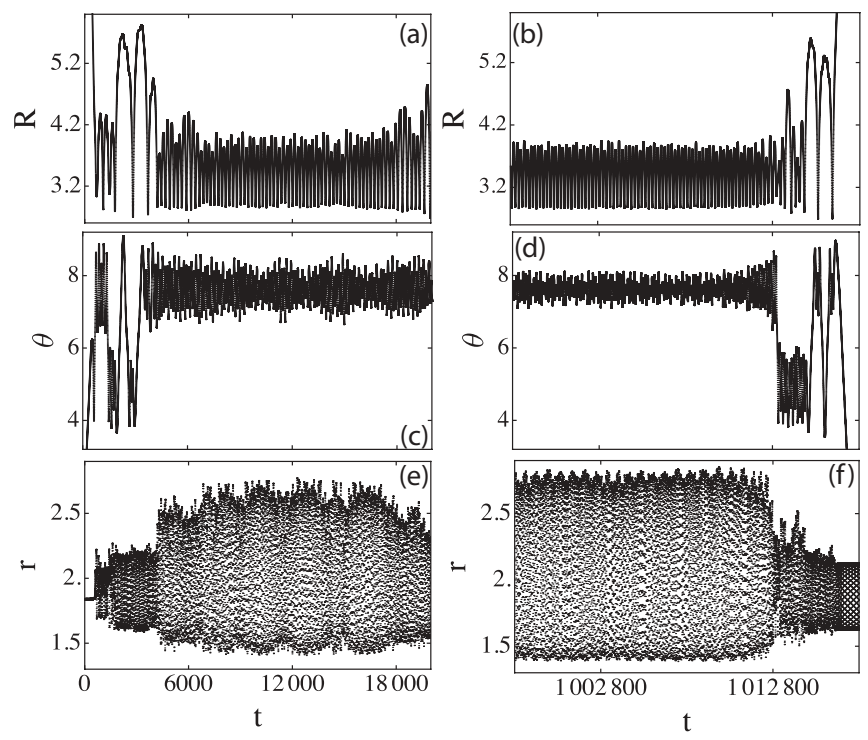

FIG. 7. The scattering dynamics of a single trajectory as it enters the reaction region $(0 \leqslant t \leqslant 20000$ d.u. $)$ and as it leaves the reaction region $(997800 \leqslant t \leqslant 1017800$ d.u.), for initial conditions $E=$ 1.231 d.u., $\psi=\pi$, and $\chi=0.994052$. (a) $R(t)$ versus $t$ (entering). (b) $R(t)$ versus $t$ (leaving). (c) $\theta(t)$ versus $t$ (entering). (d) $\theta(t)$ versus $t$ (leaving). (e) $r(t)$ versus $t$ (entering). (f) $r(t)$ versus $t$ (leaving). ( $R$, $r$, and $t$ in dimensionless units and $\theta$ in radians.)

region. The HO-Cl complex appears to be caught up in an oscillatory motion during the entire time that the $\mathrm{Cl}$ atom is in the reaction region. Plots of $p_{R}, p_{\theta}$, and $p_{r}$ versus time show variations in oscillation amplitude similar to the plots for $R(t), \theta(t)$, and $r(t)$, respectively, in Fig. 7. In Figs. 8(a), $8(\mathrm{~b})$, and $8(\mathrm{c})$, we show the power spectra of $R(t), \theta(t)$, and $r(t)$ taken for the interval of time 7000 d.u. $\leqslant t \leqslant 17000$ d.u. while $\mathrm{Cl}$ is in the reaction region. In all three cases, there are two distinct peaks embedded in a broadened background, indicting that the $\mathrm{Cl}$ is likely caught in the neighborhood of an unstable periodic orbit embedded in a chaotic sea associated with the fractal structure. The power spectra for $p_{R}(t), p_{\theta}(t)$, and $p_{r}(t)$ are the same as those shown in Figs. 8(a), 8(b), and $8(\mathrm{c})$, respectively. Note that the frequencies of the highest peaks is in the ratio 3:5:15, a further indication that the motion of the $\mathrm{HO}-\mathrm{Cl}$ system is dominated by a periodic orbit of the 3D system.

\section{MORE GENERAL INITIAL CONDITIONS}

We next examine how the scattering process changes as we vary the division of the initial HO energy between the rotation and vibration degrees of freedom. We fix the total energy at $E_{\mathrm{tot}}=1.231 \mathrm{~d}$.u. $=25000 \mathrm{~cm}^{-1}$ and the sum of the initial rotational and vibrational energies of $\mathrm{HO}$ at value $E_{\mathrm{HO}}=0.1969$ d.u. $=4000 \mathrm{~cm}^{-1}$. Thus, the initial value of $p_{R}$ is held fixed but the incident $\mathrm{Cl}$ can have a range of angular momenta, depending on the initial value of $p_{\theta}$ (in order to keep the total angular momentum of the system fixed at zero). In Figs. 9 and 10, we show the result of varying the fraction of the energy $E_{\mathrm{HO}}$ that initially goes to rotation and to vibration of the HO dimer. In Fig. 9, we show scattering dynamics when $p_{\theta}=5.92$ d.u. and vibration has amplitude in the range
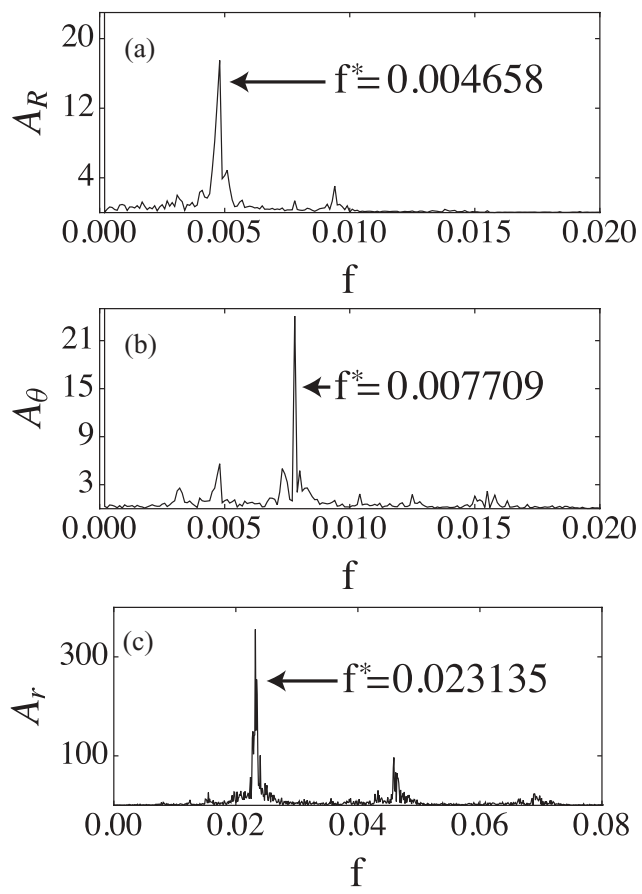

FIG. 8. Amplitudes $A(f)$ of the Fourier transform of the time series in Fig. 7, taken during the time interval 7000 d.u. $\leqslant t \leqslant$ 17000 d.u., where $f$ is the frequency. (a) $A_{R}(f)$ versus $f$ taken from time series $R(t)$ versus $t$. The highest peak is at $f^{*}=0.004658$ d.u. (b) $A_{\theta}(f)$ versus $f$ taken from time series $\theta(t)$ versus $t$. The highest peak is at $f^{*}=0.007709$ d.u. (c) (a) $A_{r}(f)$ versus $f$ taken from time series $r(t)$ versus $t$. The highest peak is at $f^{*}=0.023134$ d.u. Note that the frequencies of the highest peaks are approximately commensurate with frequency ratios 3:5:15. $\left(A_{R}, A_{r}, A_{\theta}\right.$, and $f$ in dimensionless units.)

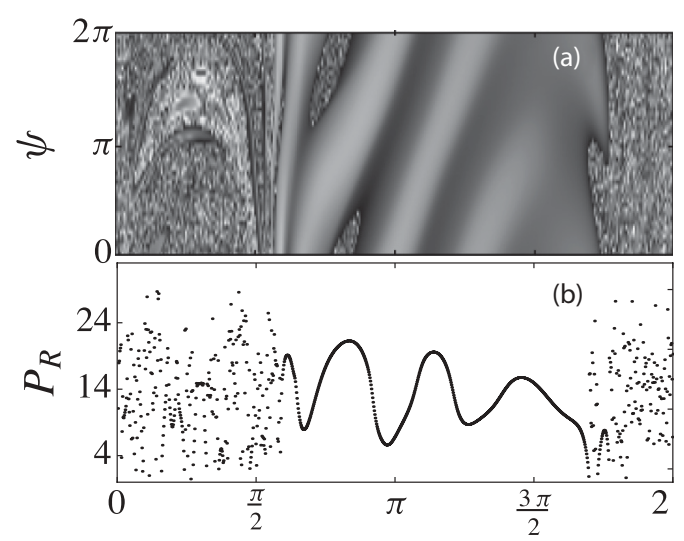

FIG. 9. The total energy is fixed at $E=1.231$ d.u. $=$ $25000 \mathrm{~cm}^{-1}$. The energy of the incident chlorine atom is fixed at $E_{\mathrm{Cl}}=1.034$ d.u. $=21000 \mathrm{~cm}^{-1}$. The remaining $E_{\mathrm{HO}}=$ 0.1969 d.u. $=4000 \mathrm{~cm}^{-1}$ is distributed between rotation and vibration of $\mathrm{HO}$ so $p_{\theta}=5.92 \mathrm{~d}$.u. and vibration has amplitude in the range 1.622 d.u. $\leqslant r \leqslant 2.134$ d.u. (a) Contour plot of scattered values of $p_{R}$ as a function of $\psi$ and $\chi$. (b) A plot of scattered values of $p_{R}$ as a function of $\chi$ for $\psi=\pi$. The grayscale in (a) ranges from 0 to 32 , with 32 the lightest shade. ( $p_{R}$ in dimensionless units and $\chi$ in radians.) 


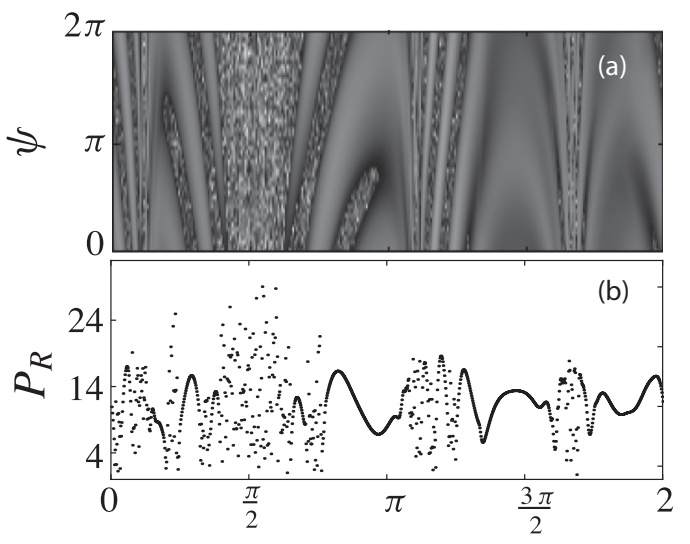

FIG. 10. The total energy is fixed at $E=1.231$ d.u. $=$ $25000 \mathrm{~cm}^{-1}$. The energy of the incident chlorine atom is fixed at $E_{\mathrm{Cl}}=1.034$ d.u. $=21000 \mathrm{~cm}^{-1}$. The remaining $E_{\mathrm{HO}}=$ 0.1969 d.u. $=4000 \mathrm{~cm}^{-1}$ is distributed between rotation and vibration of $\mathrm{HO}$ so $p_{\theta}=0.92$ d.u. and vibration has amplitude in the range 1.599 d.u. $\leqslant r \leqslant 2.159$ d.u. (a) Contour plot of scattered values of $p_{R}$ as a function of $\psi$ and $\chi$. (b) A plot of scattered values of $p_{R}$ as a function of $\chi$ for $\psi=\pi$. The grayscale in (a) ranges from 0 to 32 , with 32 the lightest shade. ( $p_{R}$ in dimensionless units and $\chi$ in radians.)

1.622 d.u. $\leqslant r \leqslant 2.134$ d.u. The fractal regions have expanded significantly but are still disconnected. Finally, in Fig. 10, we show scattering dynamics when $p_{\theta}=0.92 \mathrm{~d}$.u. and vibration has amplitude in the range 1.599 d.u. $\leqslant r \leqslant 2.159$ d.u., so the initial energy of $\mathrm{HO}$ is almost entirely vibrational. The regions of discontinuity now cover a significant portion of the initial conditions and have a clear dependence on the initial phase of the HO vibration. It is clear from Figs. 9(a) and 10(a) that the scattering dynamics is no longer even approximately independent of $\psi$ as was the case in Fig. 2. As the initial HO vibrational energy increases, the $2 \mathrm{D}$ model ceases to be a good approximation of the full 3D dynamics.

\section{CONCLUSIONS}

The qualitative behavior of the scattering dynamics of the $\mathrm{HOCl}$ molecule, just above dissociation, depends critically on the initial excitation energy of the HO dimer. If the dimer is initially close to its ground state, the scattering dynamics has only small regions of initial conditions that show fractal behavior and the scattering dynamics can be well described by the $2 \mathrm{D}$ model. If the $\mathrm{HO}$ dimer is initially in a fairly high excited state, a large portion of initial conditions show fractal behavior and the scattering functions show a clear dependence on the initial phase of the $\mathrm{HO}$ vibration. In this case, it would appear that the scattering dynamics can no longer be approximated by the 2D model.

The vibrational motion of small molecules, such as $\mathrm{HOCl}$, provides a laboratory for studying the quantum-classical correspondence (QCC) in coupled nonlinear oscillator systems. In a previous paper [17], we studied the QCC using the 2D model of the $\mathrm{HOCl}$ molecule. We found that bound states in the quantum system lie primarily on classical periodic orbits, while quasibound states are supported by unstable classical periodic orbits in the continuum. We expect that, in regimes where $\mathrm{HO}$ vibration begins to participate in the scattering process, new classes of long-lived quasibound states will be supported by unstable periodic orbits such as those found in Fig. 8. Studies of quasibound state formation, when the HO vibration has significant excitation energy, will require an analysis of the full 3D model of the HOCL molecule.

\section{ACKNOWLEDGMENTS}

The authors (Lin, Barr, and Reichl) wish to thank the Robert A. Welch Foundation (Grant No. F-1051) for support of this work. Author Jung wishes to thank CONACyT (Grant No. 79988) and DGAPA (Grant No. IN110110) for support of this work. The authors also thank Kyungsun $\mathrm{Na}$ for useful discussions.
[1] C. Jung and H. J. Scholz, J. Phys. A 20, 3607 (1987); 21, 2301 (1988).

[2] B. Rückerl and C. Jung, J. Phys. A 27, 55 (1994).

[3] The collection of articles in Chaos (Volume 3, number 4) give an overview of early developments on chaotic scattering.

[4] A. Emmanouilidou, C. Jung, and L. E. Reichl, Phys. Rev. E 68, 046207 (2003).

[5] C. Jung and A. Emmanouilidou, Chaos 15, 023101 (2005).

[6] A. Emmanouilidou and C. Jung, Phys. Rev. E 73, 016219 (2006).

[7] A. M. Barr, K. Na, L. E. Reichl, and C. Jung, Phys. Rev. E 79, 026215 (2009).

[8] Yi-Der Lin, A. M. Barr, K. Na, and L. E. Reichl, Phys. Rev. E 83, 056217 (2011).

[9] S. Wiggins, Normally Hyperbolic Invariant Manifolds in Dynamical Systems (Springer-Verlag, Berlin, 1994).
[10] C. Jung, O. Merlo, T. H. Seligman, and W. P. K. Zapfe, New J. Phys. 12, 103021 (2010).

[11] H. Waalkens, R. Schubert, and S. Wiggins, Nonlinearty 21, R1 (2008).

[12] H. Waalkens and S. Wiggins, Reg. Chao. Dyn. 15, 1 (2010).

[13] Z. Kovacs and L. Wiesenfeld, Phys. Rev. E 63, 056207 (2001).

[14] J. Weiss, J. Hauschildt, S. Yu. Grebenshchikov, R. Duren, R. Schinke, J. Koput, S. Stamatiadis, and S. C. Farantos, J. Chem. Phys. 112, 77 (2000).

[15] We thank R. Schinke for providing access to the PES used in this work.

[16] M. Joyeux, D. Sugny, M. Lombardi, R. Jost, R. Schinke, S. Skokov, and J. Bowman, J. Chem. Phys. 113, 9610 (2000).

[17] A. M. Barr, K. Na, and L. E. Reichl, Phys. Rev. A 83, 062510 (2011). 